\title{
Sensitivity enhancement in high resolution stimulated Raman spectroscopy of gases with hollow-core photonic crystal fibers
}

\author{
José Luis Doménech* and Maite Cueto \\ Molecular Physics Department, Instituto de Estructura de la Materia IEM-CSIC, Serrano 123, 28006 Madrid, Spain \\ *Corresponding author: jl.domenech@csic.es
}

Received June 28, 2013; revised August 12, 2013; accepted September 3, 2013; posted September 5, 2013 (Doc. ID 193046); published October 7, 2013

\begin{abstract}
We show the first experimental evidence of the sensitivity enhancement that can be achieved in high resolution stimulated Raman spectroscopy of gases using hollow-core photonic crystal fibers (HCPCFs). Using low power cw lasers and a HCPCF containing the gas, we have observed more than four orders of magnitude enhancement of sensitivity when compared with the cw single focus regime, and a similar sensitivity to that achieved in the more sensitive quasi-cw setups with multipass cells. (c) 2013 Optical Society of America

OCIS codes: (120.6200) Spectrometers and spectroscopic instrumentation; (290.5910) Scattering, stimulated Raman; (300.6320) Spectroscopy, high-resolution; (300.6450) Spectroscopy, Raman; (060.4005) Microstructured fibers.

http://dx.doi.org/10.1364/OL.38.004074
\end{abstract}

Hollow-core photonic crystal fibers (HCPCFs) [1] have proven to be a very efficient way to enhance nonlinear optical effects in gaseous media, due to the long interaction lengths and tight confinement of the gases and electromagnetic radiation inside the fiber core. Regarding spectroscopic applications, HCPCFs filled with methane, acetylene, ammonia, iodine, etc. have enabled new sensing devices and frequency standards, using either direct or saturated absorption spectroscopy (see, e.g., [2] and references therein.) The Raman effect (both spontaneous and stimulated) has also been exploited in this type of fiber, although with less emphasis on the spectroscopic applications. In particular, frequency conversion using the stimulated Raman effect in hydrogen has achieved near quantum-limited efficiency using HCPCF [3] , allowing frequency conversion with ultralow thresholds and making it possible to generate frequency combs spaced by combinations of vibration and rotation frequencies of the molecule. Fedotov et al. [4] have recorded an unresolved CARS spectrum of the $Q$-branch of $N_{2}$ using this type of fiber. The spontaneous Raman effect has also been used in HCPCFs [5] , showing an enhancement of several hundred times in the Raman signal. In this work, we demonstrate that high resolution stimulated Raman spectroscopy (SRS) can benefit enormously from this type of fiber, enabling the use of $\mathrm{cw}$ lasers of high spectral purity and moderate power to record high resolution Raman spectra of gases, with a sensitivity comparable to that attainable using pulsed sources.

Very succinctly, in SRS, two lasers are focused onto the sample. When the frequency difference between them matches that of a Raman-allowed transition, the high frequency laser losses power, while the low frequency laser gains it. The molecule is excited to the upper energy level of the transition closing the energy balance. Usually a pump laser and a probe laser are used and one of them is tunable. Depending on whether the probe laser is the low or the high frequency one, the techniques are named stimulated Raman gain spectroscopy and stimulated Raman loss spectroscopy (also called inverse Raman spectroscopy.) In the plane wave approximation, the fractional change of power in the probe beam can be expressed as [] $]$

$$
G \equiv\left|\frac{\delta P\left(\nu_{\text {probe }}\right)}{P\left(\nu_{\text {probe }}\right)}\right| \approx \frac{N}{h c^{2} \nu_{\text {probe }}^{3}}\left(\frac{d^{2} \sigma}{d \Omega d \nu_{\text {probe }}}\right) I\left(\nu_{\text {pump }}\right) L,
$$

where $\delta P\left(\nu_{\text {probe }}\right)$ is the change induced in the probe intensity $P\left(\nu_{\text {probe }}\right) /(\mathrm{W})$ at wavenumber $\nu_{\text {probe }}$ by the pump beam of intensity $I\left(\nu_{\text {pump }}\right) /\left(\mathrm{W} \mathrm{cm}^{-2}\right)$ at wavenumber $\nu_{\text {pump }}, N$ is the population difference between upper and lower states, $\left(d^{2} \sigma / d \Omega d \nu_{\text {probe }}\right) /\left(\mathrm{cm}^{3} \mathrm{sr}^{-1}\right)$ is the spectrally resolved differential Raman scattering cross section, and $L$ is the pathlength over which the beams interact with the medium. Integration of Eq. (1) over the interaction volume for the case of two collinear Gaussian beams with identical beam parameters yields

$$
G \approx \frac{N \pi}{h c^{2} \nu_{\text {probe }}^{2}}\left(\frac{d^{2} \sigma}{d \Omega d \nu_{\text {probe }}}\right) P\left(\nu_{\text {pump }}\right)
$$

where $P\left(\nu_{\text {pump }}\right) /\left(\mathrm{Js}^{-1}\right)$ is the pump power. This expression is independent of the beam parameters (i.e., the degree of focusing) and assumes that the focal volume of the two beams is completely embedded in the sample gas. Historically, cw SRS was the first technique developed for SRS of gases, but it soon became obvious that further enhancements in sensitivity, beyond the use of multipass refocusing cells, would only be feasible with high peak-power-pulsed sources, leading to the development of the quasi-cw stimulated Raman technique [6]. In that setup, a high power-pulsed laser with Fourier transform limited bandwidth is used as a pump beam (with peak power in the megawatt or hundreds of kilowatt range), and a low power, low noise $\mathrm{cw}$ probe laser is monitored with time-gated detection, as one of the lasers is frequency tuned. This technique remains to date the best compromise between spectral resolution and sensitivity for Raman spectroscopy of gases. According to [7] one can expect a $\sim 4000$-fold enhancement in sensitivity from quasi-cw SRS respect to cw SRS, under typical 
conditions from available laser sources. It is the purpose of this Letter to show that, using the cw SRS technique, at least the same enhancement factor can be achieved by placing the gas sample inside a HCPCF, without the added complexity and expense of a Fourier transform limited pulsed setup. Using a HCPCF corresponds to having a focus at every point along the fiber thus creating a high intensity overlap with many more molecules, with the gain of the process governed by Eq. (1).

The experiment is depicted in Fig. 1 . The probe beam is a single mode $\mathrm{Ar}^{+}$laser, actively frequency stabilized and locked to the ${ }^{127} I_{2} a_{3}$ hyperfine component of the $P(13)$ 43-0 transition, known with an accuracy $\sim 0.1 \mathrm{MHz}$ [8] . When the stabilization loop is closed, the laser frequency has a residual frequency jitter less than $1 \mathrm{MHz}$ and a longterm stability of the same order of magnitude. The pump beam is a tunable single mode ring dye laser with commercial stabilization electronics (residual jitter less than $3 \mathrm{MHz}$ ), whose frequency is measured with a high accuracy $(10 \mathrm{MHz})$ commercial wavemeter, calibrated with the stabilized $\mathrm{Ar}^{+}$laser. The dye laser was operated with Rhodamine 110. Both lasers are overlapped with a dichroic mirror and directed onto the fiber. The frequency stabilization and scan control box in Fig. 1 comprises a Doppler-free polarization spectroscopy setup for the $\mathrm{Ar}^{+}$ laser frequency lock, the frequency stabilization and scan control system of the ring dye laser and the wavemeter.

The HCPCF is a $1 \mathrm{~m}$ long piece of HC-532-02 fiber from NKT Photonics [9]. It has a core diameter of $4.8 \pm 0.5 \mu \mathrm{m}$, a mode field diameter of $4 \pm 1 \mu \mathrm{m}$, and a numerical aperture of $\sim 0.2$. The best coupling efficiency into the fiber has been achieved using a $\times 2$ Keplerian telescope in the path common to both beams and $\mathrm{a} \times 10$ plan-achromat microscope objective with $0.25 \mathrm{NA}$ and $10.3 \mathrm{~mm}$ working distance. At the fiber output, a $15 \mathrm{~mm}$ focal lens collimates the beams before sending them to the detector. We have used powers of $40 \mathrm{~mW}$ in the pump laser and $4 \mathrm{~mW}$ in the probe laser, both measured before the focusing objective. The transmitted pump power, measured after the fiber and collimating lens, was $15-20 \mathrm{~mW}$, somewhat less than $50 \%$, for the pump beam. This is less than expected from the transmission losses of the fiber as specified by the manufacturer. The intensity distribution at the fiber output shows a mode pattern with a central bright spot, which carries 90\% of the transmitted power,

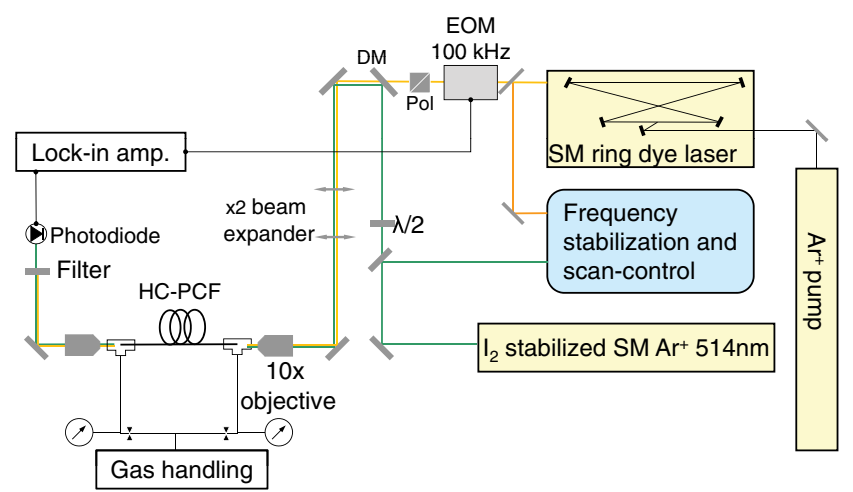

Fig. 1. Schematic of the experimental setup. Pol, polarization beamsplitter; DM, dichroic mirror; EOM, electro-optic modulator. surrounded by side lobes whose relative intensity is very sensitive to coupling alignment. This complex mode structure, frequent in these fibers, and the low total transmission, evidence that we are exciting more fiber modes besides the fundamental, and that there is room for improvement at the light coupling stage. It must be noted that SRS is a self-phase matched process, so no interferences due to a mismatch of the propagation constant of different modes are expected, and, we have no evidence of them.

In the path of the $\mathrm{Ar}^{+}$laser there is a half-wave plate in order to make the polarizations of both beams parallel. There are two reasons for it. (1) Although the fiber is not polarization maintaining by design, the residual birefringence clearly establishes a preferred plane of transmission, with much higher losses when the polarizations are perpendicular to it. (2) With parallel polarizations, one observes both the isotropic and the anisotropic components of the Raman polarizability tensor, leading to larger signals.

The pump laser is amplitude modulated at $100 \mathrm{kHz}$ with an electro-optic modulator (EOM) and a polarizer. The beams exiting the fiber are sent to a laser cleanup filter designed for $514.5 \mathrm{~nm}$, with OD $>6$ at the frequency of the pump laser. After the filter, the probe beam is detected by a silicon photodiode, coupled to a transimpedance amplifier and then to a lock-in amplifier. The latter is locked to the modulation frequency of the EOM and discriminates the probe power changes which are in phase with the pump beam amplitude modulation, rendering the Raman spectrum of the sample as the pump laser is scanned. The frequency of the Raman spectrum is calculated directly as the difference between the (known) frequency of the probe laser and the wavemeter reading, which takes just $1 \mathrm{~ms}$.

In order to couple the light and the gas sample in and out of the HCPCF, two small heads have been designed with optical windows and vacuum connections. The final assembly is clamped to a precision XYZ stage, which allows for fine movement of the input fiber tip with respect to the lasers foci. The exit end alignment is much less critical, since it only has to ensure that the probe beam fills the sensitive area of the photodiode.

Several groups (e.g., $[10,11])$ have studied the gas transport dynamics in these narrow capillaries. We have done a numerical integration of the Knudsen equation, without simplification to the limiting cases of viscous or molecular flow. We have used Knudsen's equation as given in [12]

$$
\frac{\partial p}{\partial t}=\frac{\partial}{\partial x}\left[\left(\frac{d^{2} p}{32 \eta}+\frac{d \bar{v}}{3} \frac{1+\sqrt{8 / \pi} d p /(\eta \bar{v})}{1+1.23 \sqrt{8 / \pi} d p /(\eta \bar{v})}\right) \frac{\partial p}{\partial x}\right],
$$

where $p$ is the instantaneous pressure at time $t$ and distance $x$ along the fiber, $d$ is the core diameter, $\bar{v}$ the mean velocity of the gas molecules at the temperature of the experiment, and $\eta$ the viscosity. Summarizing our results, we estimate to have the core of the fiber completely evacuated (pressure below $10^{-2}$ mbar at any point in the core) in less than $40 \mathrm{~min}$, and a stable working pressure in about $20 \mathrm{~min}$. 
Regarding the spectral coverage of the present setup, if we accept $50 \%$ transmission losses, the transmission window, according to the manufacturer, for a $1 \mathrm{~m}$ length of fiber is $\sim 560-486 \mathrm{~nm}\left(\sim 17860-20120 \mathrm{~cm}^{-1}\right)$. Since both beams have to be transmitted efficiently, this means that using the different lines from the $\mathrm{Ar}^{+}$laser (514, 496, and $488 \mathrm{~nm}$ ) and Rhodamine 110 as dye, we can access Raman frequencies between 1000 and $2600 \mathrm{~cm}^{-1}$. With different lasers and fibers, not available for this work, this frequency range can be extended. In particular, with lasers emitting at very close frequencies, very small Raman shifts, corresponding to pure rotation transitions, can be reached, while the upper frequency limit will stay limited by the transmission bandwidth of the fiber.

The first spectrum we have used as a test is that of the $Q$-branch of the $\nu_{1}$ component of the $\nu_{1} / 2 \nu_{2}$ Fermi dyad of $\mathrm{CO}_{2}$ (see e.g., [13] for a description of the energy levels.) As pointed out in [14], this vibrational $Q$-branch is remarkably narrow, with all rovibrational components piled up, due both to the small change in the $B$ rotational constant between the upper and lower vibrational levels, and to the onset of line mixing, which occurs when the inelastic collision frequency exceeds the separation between the $Q$-branch components. As a result, there is one single and narrow line in the spectrum, which contains the intensity of all rovibrational components. Figure 2 shows the spectrum obtained with $930 \mathrm{mbar}$ of pure $\overline{\mathrm{CO}}_{2}$ in the fiber, along with a $\times 100$ intensity blow up to expose the signal to noise $(\mathrm{S} / \mathrm{N})$ ratio. The recording was made at scan speed $0.01 \mathrm{~cm}^{-1} \mathrm{~s}^{-1}$ with a lock-in time constant of $30 \mathrm{~ms}$. The trace at the center of the figure is the same $\times 100$ expanded trace after subtraction of the slowly changing baseline outside of the line. The origin of these fluctuations seems related to the stability of the coupling stage. It is not reproducible from one scan to the next, and a worst case peak-to-peak value is also shown in Fig. 2. The attained signal-to-noise ratio, after subtraction of this background, is $\sim 10000$. (Before background correction the $\mathrm{S} / \mathrm{N}$ takes values between 10,000

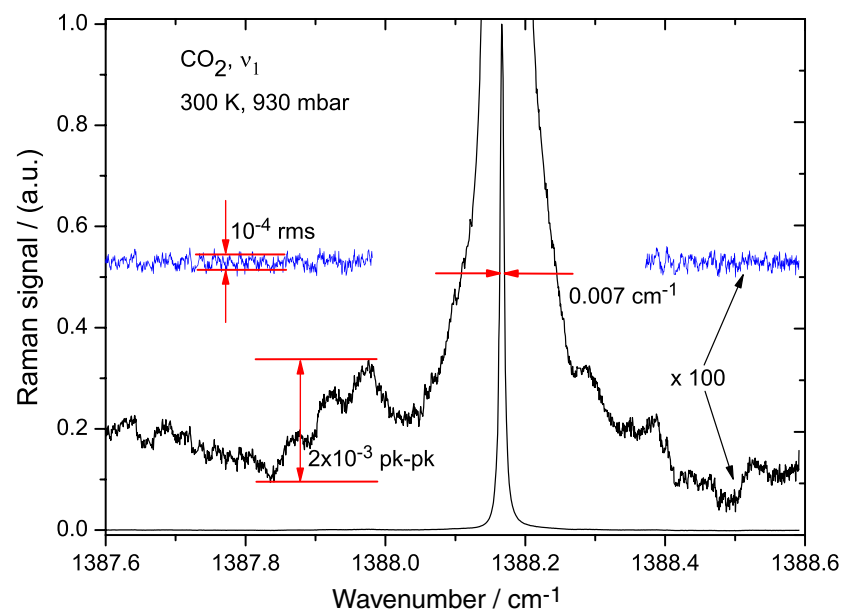

Fig. 2. SRS spectrum of the $\nu_{1}$ band of $\mathrm{CO}_{2}$ in a HCPCF. The spectrum (lower trace) has been normalized to a peak intensity of one. The two upper traces are in a $\times 100$ expanded scale. The trace at the center (blue) shows the noise level after subtraction of the slowly changing background and has been offset for clarity. and 2500 in a six linewidths interval depending on the particular part of the baseline considered.)

In [14], the authors recorded the same line with a cw SRS setup. This reference gives enough experimental details, so that a fair comparison can be made: they used a multipass refocusing cell to enhance the signal by a factor of $\sim 20$, and a polarization method to achieve sensitivity within $\sim 5-6$ times the shot noise limit. They quote $\mathrm{S} / \mathrm{N}=200$, using $200 \mathrm{~mW}$ pump power (versus $\sim 40 \mathrm{~mW}$ in our case), with a detection time constant of $1 \mathrm{~s}$. Once the noise dependence with the square root of the detection bandwidth and the difference in pump powers are factored out, our setup has a sensitivity 1400 times higher than that of [14]. Besides, if we take into account the $\times 20$ gain due to the multiple reflection cell used in [14], then the enhancement in sensitivity over the single focus regime reaches the remarkable factor of $\times 28000$ when using a $\sim 1 \mathrm{~m}$ long HCPCF as a cell. In [13] there is no mention to any background fluctuation correction, but we believe that our quoted $\mathrm{S} / \mathrm{N}$ of 10,000 is a good estimate to compare the sensitivity of the techniques, since the "period" of these fluctuations extends over several linewidths. Furthermore, their origin seems to be of technical origin, particular to the stability of our setup, and not inherent to the technique.

Figure 3 shows the spectrum of the lower frequency component $\left(2 \nu_{2}\right)$ of the same Fermi dyad of $\mathrm{CO}_{2}$. The structure of this $Q$-branch is quite different, since the $\Delta B$ value allows the resolution of the individual rovibrational components. The Raman cross section of this branch is about the same of that of $\nu_{1}$, leading to much lower peak intensities, and besides, we have reduced the pressure to 29 mbar in order to decrease pressure broadening and prove the capabilities of the technique as a high resolution spectroscopic tool. The spectrum shown is the average of 16 consecutive scans taken at a $0.01 \mathrm{~cm}^{-1} \mathrm{~s}^{-1}$ scan rate with a detection time constant of $30 \mathrm{~ms}$. The reader is referred to Fig. 6(b) of [15] for a comparison. Note that in [15], the authors use a quasi-cw setup (outlined above as a pulsed pump beam and time gated detection) and a multipass cell with a gain of $\times 24$. A direct comparison is more difficult in this case,

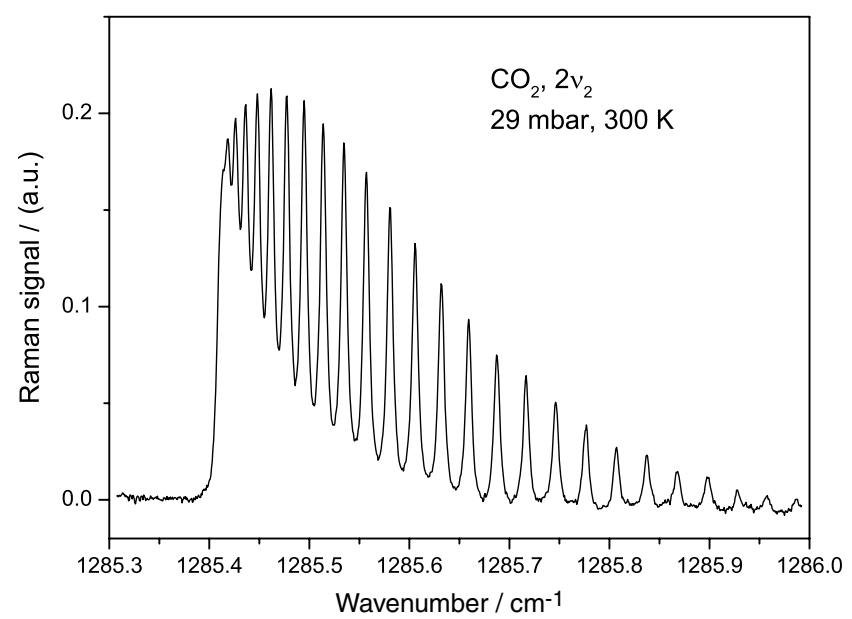

Fig. 3. Low pressure SRS spectrum of the $Q$-branch of the $2 \nu_{2}$ component of the Fermi dyad of $\mathrm{CO}_{2}$. The time constant is $30 \mathrm{~ms}, 16$ averaged scans at $0.01 \mathrm{~cm}^{-1} / \mathrm{s}$. 
since there is no information in [15] about the total recording time, the number of averaged shots, pulse energies, etc. However, from our own experience with the quasi-cw technique [16], a 10 shot exponential average with a $10 \mathrm{~Hz}$ repetition rate pump laser and a scan speed of $0.001 \mathrm{~cm}^{-1} \mathrm{~s}^{-1}$ are common, so the total recording time (although probably somewhat longer in our case) is of the same order of magnitude. A quantitative comparison is not easy, but, from inspection, the $\mathrm{S} / \mathrm{N}$ ratios are very similar, to say the least, with our spectrum showing a smoother intensity envelope. Figure 6(a) of [14], shows the same spectrum in a single pass configuration, making the enhancement of sensitivity over the single focus regime much more apparent, even with a pulsed pump.

Regarding the repeatability of the experiments, we find that, as far as intensities are concerned, it is limited by the coupling efficiency of the pump beam, which can exhibit changes of up to $25 \%$ from day to day, and whose compensation has not yet been attempted. From the individual scans that have been averaged to obtain the spectrum in Fig. 3, we have checked that the frequency scale is reproducible to better than the $10 \mathrm{MHz}$ manufacturerstated accuracy of the wavemeter. It is worth noting that in neither experiment we have seen any evidence of interference fringes.

The observed linewidths [full width at half-maximum (FWHM)] in the spectrum have several contributions. (1) The combined linewidth of the lasers, which is dominated by that of the ring dye laser, is $\sim 3 \mathrm{MHz}\left(10^{-4} \mathrm{~cm}^{-1}\right)$. (2) The Doppler width, which depends on the frequency of the molecular transition, the molecule mass, and the temperature. For $\mathrm{CO}_{2}$ at $1285 \mathrm{~cm}^{-1}$ it is $2.4 \times 10^{-3} \mathrm{~cm}^{-1}$ (73 MHz). (3) Pressure broadening, contributing with a Lorentzian component with a width proportional to the pressure. For $\mathrm{CO}_{2}$ at $29 \mathrm{mbar}$ an average value for low- $J$ lines is $6.6 \times 10^{-3} \mathrm{~cm}^{-1}(100 \mathrm{MHz})$ [15]. (4) Transit time broadening, which assuming a Gaussian profile for both lasers with $1 / e^{2}$ intensity diameter equal to the mode field diameter $\left(d_{\mathrm{mf}}\right)$, can be approximated by $\Delta \nu_{\mathrm{tt}} \approx 0.75 \bar{v} / d_{\mathrm{mf}} ;$ for $\mathrm{CO}_{2}$ in our conditions, $\Delta \nu_{\mathrm{tt}} \approx$ $72 \mathrm{MHz}\left(2.4 \times 10^{-3} \mathrm{~cm}^{-1}\right)$. A quick line profile analysis of Fig. 3 spectra yields Lorentzian lines with $\sim 0.007 \mathrm{~cm}^{-1}$ FWHM, evidencing that pressure broadening dominates the spectral resolution in this particular example. Pressure broadening can be reduced by working at lower pressures, and the lasers linewidth does not contribute significantly, so Doppler and transit time broadening limit the spectral resolution attainable with this configuration. Since both mechanisms have the same dependence on molecular mass and temperature, only the use of fibers with larger core diameter can decrease transit time broadening to the point where the resolution would be at the Doppler limit.

In summary, we have shown that using a HCPCF as sample cell in cw high resolution gas-phase SRS can provide a sensitivity enhancement of more than four orders of magnitude as compared with the single focus regime. This enhancement is comparable to that attained using pulsed pump lasers and a multipass refocusing cell.
Moreover, this work is just a first proof of the technique, and there are many developments that can still be made, which will likely improve over these results. (1) Splicing the input end of the fiber to a solid SM fiber, which will help increase the coupling stability at the launching stage (at the expense of longer filling and evacuation times). (2) Using a Kagomé-type fiber [17] with larger bore and a broader transmission window in the visible, which will overcome the transit time limit to the spectral resolution, and the upper frequency cutoff for the Raman frequencies. (3) Increasing the cw pump power. Furthermore, trying the quasi-cw approach and a HCPCF (trading off some spectral resolution) is a very attractive possibility. The results presented in this Letter show the enormous potential that using HCPCF have in Raman spectroscopy of gases.

This work has been funded by the Spanish MINECO through grants CSD2009-00038, FIS2009-08069, and FIS2012-38175. M.C. acknowledges financial support from EU-FEDER funds through a CSIC JAE-Tec contract. We wish to express our gratitude to Dr. J.-D. Ania for his help and advice in the initial steps of this work, and to him and to Dr. J. Santos for reading this Letter. Our skillful technicians, Mr. M. A. Moreno and Mr. J. R. Rodriguez, are also gratefully acknowledged.

\section{References}

1. P. Russell, Science 299, 358 (2003).

2. A. M. Cubillas, J. Hald, and J. C. Petersen, Opt. Express 16, 3976 (2008).

3. F. Couny and F. Benabid, J. Opt. A 11, 103002 (2009).

4. A. B. Fedotov, S. O. Konorov, V. P. Mitrokhin, E. E. Serebryannikov, and A. M. Zheltikov, Phys. Rev. A 70, 1 (2004).

5. M. P. Buric, K. P. Chen, J. Falk, and S. D. Woodruff, Appl. Opt. 47, 4255 (2008).

6. P. Esherick and A. Owyoung, Advances in Infrared and Raman Spectroscopy, R. J. H. Clark and R. E. Hester, eds. (Heyden, 1982), Vol. 9, pp. 130-187.

7. A. Owyoung, Chemical Applications of Nonlinear Raman Spectroscopy, A. B. Harvey, ed. (Academic, 1981), pp. 281-320.

8. T. J. Quinn, Metrologia 40, 103 (2003).

9. NKT Photonics, "HC-532-02 hollow core photonic bandgap fiber," http://www.nktphotonics.com/files/files/HC-532.pdf.

10. J. Henningsen and J. Hald, Appl. Opt. 47, 2790 (2008).

11. I. Dicaire, J. Beugnot, and L. Thévenaz, Appl. Opt. 49, 4604 (2010).

12. E. S. Creutz and L. R. Zumwalt, J. Appl. Phys. 33, 2883 (1962).

13. G. Herzberg, Molecular Spectra and Molecular Structure: Infrared and Raman Spectra of Polyatomic Molecules (Krieger, 1991), Chap. 2, p. 217.

14. J. Baran, A. Grofcsik, and W. J. Jones, Mol. Phys. 45, 1291 (1982).

15. R. Saint-Loup, B. Lavorel, G. Millot, C. Wenger, and H. Berger, J. Raman Spectrosc. 21, 77 (1990).

16. J. Santos, P. Cancio, J. L. Domenech, J. Rodriguez, and D. Bermejo, Laser Chem. 12, 53 (1992).

17. F. Couny, F. Benabid, and P. S. Light, Opt. Lett. 31, 3574 (2006). 